\title{
Ultrasonografía en dos protocolos de superovulación en donantes Braford del nordeste argentino
}

\author{
Yostar, E.J.'; Capellari, A. ${ }^{2}$; Stahringer, R.C. ${ }^{3}$; Mujica, I.F.; Munar, C.J. ${ }^{4}$
}

${ }^{1}$ Alumno de Maestría Prod. Anim. Subtrop., Fac.Cs.Vet.(FCV), Univ.Nac.del Nordeste (UNNE), Corrientes, Argentina. ${ }^{2}$ Cátedra Prod. Bov. FCV-UNNE. ${ }^{3}$ INTA Colonia Benítez, Chaco, Argentina. ${ }^{4}$ Centro Biotecn. Reprod. Bov. "Munar y Asociados” (La Plata, Buenos Aires). E-mail: jonayostar@gmail.com

\begin{abstract}
Resumen
Yostar, E.J.; Capellari, A.; Stahringer, R.C.; Mujíca, I.F.; Munar, C.J.: Ultrasonografía en dos protocolos de superovulación en donantes Braford del nordeste argentino. Rev. vet. 29: 1, 26-30, 2018. La reproducción de animales de mayor valor genético ha llevado a desarrollar distintas biotecnologías, como la transferencia de embriones. Los tratamientos de superovulación producen respuestas muy variables sobre todo en las razas sintéticas. El objetivo fue evaluar la respuesta superovulatoria, en vacas donantes Braford del nordeste argentino, mediante el seguimiento ecográfico de los ovarios. Se trabajó con 60 donantes, asignadas aleatoriamente a dos tratamientos o protocolos ( $\mathrm{T} 1: \mathrm{n}=30$ y T2: $n=30$ ); examinándose ecográficamente 6 por tratamiento, el día 0 para determinar ciclicidad, al comienzo de las aplicaciones de FSH (T1=día 3 y T2=día 4), en el control de celos (T1=día 7 y T2=día 8) para determinar respuesta superovulatoria y en la colecta $(\mathrm{T} 1=$ día 14 y $\mathrm{T} 2=$ día 15$)$. Las variables cualitativas, de distribución binomial, se analizaron mediante tablas de contingencia para el cálculo de frecuencias absolutas y relativas y prueba de $\operatorname{chi}^{2}(\mathrm{p}<0,05)$. El número de embriones transferibles logrado fue de $6,85 \pm 0,77$; embriones degenerados de $1 \pm 0,12$ y ovocitos sin fertilizar de 5,32 $\pm 1,01$. La ultrasonografía en la colecta no mostró diferencias en los cuerpos lúteos totales $(12,6$ vs 12,06), sin embargo fueron superiores en el ovario derecho $(7$ y 6,66$)$ respecto al izquierdo $(5,6$ y 5,4$)$ en $\mathrm{T} 1$ y T2, respectivamente. La prueba de independencia no estableció evidencia estadísticamente significativa. Concluimos que el adelanto en la superestimulación no genera mejoras en la respuesta, pudiendo utilizarse como una alternativa en la producción de embriones, sin afectar la cantidad y calidad de estructuras ováricas a la ultrasonografía, acortando los tratamientos tradicionales.
\end{abstract}

Palabras clave: bovinos, onda folicular, superovulación, ultrasonografía.

\begin{abstract}
Yostar, E.J.; Capellari, A.; Stahringer, R.C.; Mujíca, I.F.; Munar, C.J.: Ultrasonography in two superovulation protocols in Braford donors of the Argentinean northeast. Rev. vet. 29: 1, 26-30, 2018. Reproducing genetic merit animals led the development of different biothecnologies such as "embryo transfer". Superovulation treatments produce variable responses especially in synthetic cow breeds. The aim of the present work was to evaluate superovulatory response in Braford donors from the Argentinean northeast region trough monitoring ovaries by ultrasound. The study used 60 donors randomly assigned for two protocol treatments (T1: $n=30$ and $T 2: n=30$ ), being examined only 6 animals per treatment on day 0 to determine cyclicity, then before injecting FSH ( $11=$ day 3 and $\mathrm{T} 2=$ day 4$)$, for estrus control and to determine superovulatory response ( $\mathrm{T} 1=$ day 7 and $\mathrm{T} 2=$ day 8$)$, and finally at embryo collection ( $\mathrm{T} 1=$ day 14 and $\mathrm{T} 2=$ day 15$)$. Qualitative variables (with binomial distribution), were analyzed using contingency tables to calculate absolute and relative frequencies and $\mathrm{chi}^{2}$ test $(\mathrm{p}<0.05)$. The number of transferable embryos achieved was $6.85 \pm 0.75$, degenerate embryos were $1 \pm 0.12$, and unfertilized oocytes were $5.32 \pm 1.01$. Ultrasonography in embryo collection did not show differences in total corpora lutea (12.6 vs 12.06), however, they were higher in right ovary (7 and 6.66) than in the left (5.6 and 5.4) in T1 and T2, respectively. The independence test did not established statistic significant evidence. We conclude that the great advances on superstimulation do not generate significant improvements on treatment responses, but it can be used as an alternative in embryo production, without affecting quantity and quality of ovarian structures at ultrasonography; even shortening the traditional treatments.
\end{abstract}

Key words: bovine, follicular wave, superovulation, ultrasonography. 


\section{INTRODUCCIÓN}

La reproducción de animales de alto valor genético ha llevado a desarrollar distintas biotecnologías que permiten obtener mayor cantidad de descendientes en el menor tiempo posible. Para lograr tal objetivo se pueden utilizar técnicas como la transferencia de embriones $^{2}$, que consiste en seleccionar una hembra donante genéticamente superior, a la cual se le sincroniza el estro por medio de tratamientos hormonales.

A continuación, se induce una ovulación múltiple con la ayuda de gonadotrofinas, técnica por la cual se procura que la donante conduzca varios folículos hasta el estado ovulatorio para obtener un mayor número de ovocitos viables, que serán fecundados por medio de la liberación de material seminal a nivel uterino (inseminación artificial) ${ }^{3}$. Finalmente, los embriones serán recuperados del útero el día 7 post-inseminación, cuando aún no se han implantado en la superficie uterina ${ }^{11}$.

Para interpretar cabalmente los tratamientos superovulatorios, es importante recordar que el ciclo estral bovino se caracteriza por la presencia de 2 ó 3 ondas foliculares, cada una de las cuales se distingue por una etapa de reclutamiento de 20 a 30 folículos, seguida por la selección y crecimiento de un folículo dominante y posterior atresia en caso de no acontecer la luteólisis ${ }^{16}$. Cada onda folicular tiene una duración de alrededor de 8-10 días, dependiendo de la presencia del cuerpo lúteo ${ }^{1}$.

La dinámica folicular ha sido estudiada a través de diferentes técnicas, siendo las más aplicadas la ultrasonografía y la medición de niveles hormonales, mostrando variaciones que dependen de la genética, el estado fisiológico, el clima, la nutrición y el sistema de producción. El estudio de la dinámica folicular tiene como finalidad mejorar el conocimiento de los principios fisiológicos que rigen la producción de óvulos y hormonas, así como su relación con el comportamiento reproductivo ${ }^{14}$.

Durante cada ciclo de crecimiento folicular, un folículo dominante se desarrolla más rápidamente y suprime el crecimiento de otros más pequeños. Cuando el dominante se desarrolla durante la última ola de cada ciclo estral se produce la ovulación ${ }^{24}$. Informes recientes sugieren que la presencia o ausencia de un folículo dominante puede afectar los tratamientos superovulatorios. El examen ultrasonográfico diario ha demostrado que las respuestas superovulatorias se reducen en animales tratados en presencia de un folículo dominante, comparados con animales sin folículos dominantes al inicio del tratamiento superovulatorio ${ }^{15,24}$.

Con la aplicación de hormonas como FSH, GnRH, estrógenos $(1-2,5 \mathrm{mg})$, progesterona $(50 \mathrm{mg})$ y LH (o hCG), se ha logrado evitar el efecto negativo del folículo dominante y así poder iniciar el tratamiento superovulatorio en cualquier etapa del ciclo estral. La utilización de dichas hormonas no sólo elimina el folículo dominante sino que además permite sincronizar las ondas foliculares y la ovulación, permitiendo por ende realizar inseminación a tiempo fijo ${ }^{5,6}$.

Los tratamientos superovulatorios disponibles se caracterizan por provocar respuestas extremadamente variables en todas las categorías, por lo cual es imposible estimar el número de embriones que se recuperan por donante ${ }^{8,13}$. La FSH tiene una vida media corta, por lo cual requiere realizar frecuentes aplicaciones para inducir superovulación; dos aplicaciones diarias han resultado en una mayor respuesta superovulatoria que la obtenida con una sola aplicación ${ }^{6,19,26}$.

En base a lo expuesto, referente a la dinámica folicular y al inicio de una nueva onda folicular, como así también fundamentados en un ensayo preliminar donde se realizó un tratamiento superovulatorio comenzando las aplicaciones de FSH el día 3, obteniendo resultados superiores a los logrados hasta ese momento, se propone comenzar la superestimulación el día 3 y/o 4 después la aplicación de $17 \beta$-estradiol y progesterona.

El objetivo de este estudio fue evaluar la respuesta a dos tratamientos superovulatorios en donantes Braford del nordeste argentino, mediante ultrasonografía y examen de las estructuras recuperadas en la colecta.

\section{MATERIAL Y MÉTODOS}

Lugar de trabajo. El experimento se llevó a cabo en establecimientos de la región nordeste (NEA) de la República Argentina.

Animales, variables dependientes. Los animales sujetos al ensayo pertenecieron a la raza Braford, utilizados como donantes de embriones en cabañas del NEA adheridas a un Centro Biotecnológico de Reproducción Bovina (Munar y Asociados S.A.). Se trabajó con 60 donantes, asignadas aleatoriamente a dos tratamientos superovulatorios (T1: $n=30$ y T2: $n=30$ ), evaluándose la respuesta superovulatoria mediante ultrasonografía a un subgrupo de 6 donantes por tratamiento.

Los tratamientos se iniciaron con el control de las ondas foliculares y del folículo dominante, comenzando ambos el día 0 a las 08:00 h con dispositivos intravaginales impregnados con progesterona CIDR ${ }^{\circ}(1,9$ $\mathrm{g}$ de progesterona, Lab.Zoetis, Argentina) $+100 \mathrm{mg}$ de Progesterona ${ }^{\circledR}$ (Lab. Río de Janeiro, Argentina) +5 $\mathrm{mg}$ de 17- $\beta$ estradiol ${ }^{\circledR}$ (Lab.Río de Janeiro, Argentina) (P4+17ße+DIB).

En T1 el día 3 comenzó la superovulación con FSH Folltropin-V® (400 mg NIH-FSH-Pl; Vetrepharm, Canadá Inc.), 8 aplicaciones totales IM a dosis decrecientes, 2 aplicaciones diarias, 08:00 y 18:00 h (320 mg totales). En el día 5, además de la FSH, se aplicaron 150 $\mu \mathrm{g}(2 \mathrm{ml})$ de prostaglandina sintética Acceleration-D® (Lab. Vabriela, Argentina). El día 6 se retiró el dispositivo intravaginal y el día 7 se detectó la presencia de celos por la mañana $(08: 00 \mathrm{~h})$ y por la tarde $(18: 00 \mathrm{~h})$,

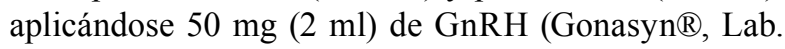
Syntex, Argentina) y realizando inseminación artificial con dos dosis (pajuelas) de semen, repitiéndose el día 8 
(08:00 h) con una sola dosis. El día 14 se llevó a cabo la colecta de embriones. A continuación se esquematizan los protocolos correspondientes.

\section{Tratamiento 1}

\begin{tabular}{c|ccccccc|c}
\hline $\begin{array}{c}\text { P4+17ße+DIB } \\
\text { 08:00 hs }\end{array}$ & FSH 08:00 hs & FSH 08:00 hs & FSH 08:00 hs & FSH 08:00 hs & 08:00 hs & \multicolumn{2}{c}{$08: 00 \mathrm{hs}$} & \multicolumn{1}{c}{ Colecta 08:00 hs } \\
\hline Día 0 & Día 3 & Día 4 & Día 5 & Día 6 & Día 7 & Día 8 & Día 14 \\
\hline & FSH 18:00 hs & FSH 18:00 hs & FSH 18:00 hs & FSH 18:00 hs & GnRH 18:00 hs \\
& & & PGF 18:00 hs & & IATF $\times 2$ dosis \\
\hline
\end{tabular}

y error estándar. Las variables fueron las estructuras totales, subdivididas en: embriones transferibles, embriones degenerados y ovocitos sin fertilizar.

Debido a que las variables estudiadas por ultrasonografía toman valores alternativos presentando distri-
En T2, la superestimulación comenzó el día 4 y de esta forma toda la secuencia del tratamiento se atrasó $24 \mathrm{~h}$ respecto a $\mathrm{T} 1$.

\section{Tratamiento 2}

\begin{tabular}{|c|c|c|c|c|c|c|c|}
\hline $\mathrm{P} 4+17 \beta \mathrm{e}+\mathrm{DIB}$ & & & PGF 08:00 hs & Retirar DIB & Control Celo & IATF $\times 2$ dosis & \\
\hline 08:00 hs & FSH 08:00 hs & FSH 08:00 hs & FSH 08:00 hs & FSH 08:00 hs & 08:00 hs & 08:00 hs & Colecta 08:00 hs \\
\hline Día 0 & Día 4 & Día 5 & Día 6 & Día 7 & Día 8 & Día 9 & Día 15 \\
\hline & FSH 18:00 hs & FSH 18:00 hs & $\begin{array}{l}\text { FSH 18:00 hs } \\
\text { PGF 18:00 hs }\end{array}$ & FSH 18:00 hs & $\begin{array}{l}\text { GnRH 18:00 hs } \\
\text { IATF } \times 2 \text { dosis }\end{array}$ & & \\
\hline
\end{tabular}

Para determinar ciclicidad, se realizó ultrasonografía ovárica el día 0 del tratamiento, al comienzo de las aplicaciones de FSH (días 3 en T1 y 4 en T2), identificando y contabilizando folículos de 1-3 mm y de 3-6 $\mathrm{mm}$. También se realizó el día de control de celos (día 7 en T1 y día 8 en T2), para determinar respuesta superovulatoria (folículo dominante) y día de la colecta (día 14 en T1 y día 15 en T2), contabilizándose cuerpos lúteos $\mathrm{y}$ otras estructuras.

\section{Distribución de las ecografías en T1 y T2}

\begin{tabular}{|c|c|c|c|c|c|c|c|}
\hline ECO 08:00 hs & \multicolumn{1}{c}{ ECO 08:00 hs } & \multicolumn{2}{c}{ ECO 08:00 hs } \\
\hline Día 0 & Día 3 & Día 4 & Día 5 & Día 6 & Día 7 & Día 8 & Día 14 \\
\hline ECO 08:00 hs & ECO 08:00 hs & \multicolumn{5}{c|}{ ECO 08:00 hs } & ECO 08:00 hs \\
\hline Día 0 & Día 4 & Día 5 & Día 6 & Día 7 & Día 8 & Día 9 & Día 15 \\
\hline
\end{tabular}

Las donantes utilizadas fueron clínicamente sanas y se manejaron bajo las mismas condiciones de alimentación, sanidad y manejo.

El semen fue producido en centros de inseminación certificados por SENASA y por la Cámara Argentina de Biotecnología de la Reproducción e Inseminación Artificial (CABIA, Argentina) o por organizaciones equivalentes de los países de origen para semen importado.

Los embriones fueron obtenidos mediante el método no quirúrgico a circuito cerrado de flujo discontinuo $9,12,17$, y luego fueron clasificados según las normas de la International Embryo Transfer Society (IETS) ${ }^{25}$.

Diseño experimental, estadística. El diseño fue completamente aleatorizado (DCA) y cada una de las 12 donantes evaluadas por ultrasonografía fueron consideradas como unidades experimentales. Se calcularon las estadísticas descriptivas: media aritmética bución binomial, se utilizaron tablas de contingencia para el cálculo de las frecuencias absolutas y relativas y la prueba de $\mathrm{chi}^{2}(\mathrm{p}<0,05)$ mediante el software Infostat/Profesional bajo entorno Window ${ }^{7}$.

\section{RESULTADOS Y DISCUSIÓN}

Los resultados para embriones transferibles $(6,85 \pm 0,77)$ fueron inferiores a lo informado por otros autores, de 7,8 $\pm 0,3$ en razas sintéticas Braford y Brangus, y 7,5 $\pm 0,6$ en Bonsmara ${ }^{21} ; 7,6 \pm 0,7$ en Bonsmara ${ }^{28}$ y $7,7 \pm 1,1$ en Brangus ${ }^{29}$, aunque fueron superiores a $5,7 \pm 0,3^{21}$ y $5,7 \pm 0,6$ en donantes Aberdeen Angus ${ }^{27}$. No obstante, fueron similares a los logrados en la raza Simmental ${ }^{21}(6,8 \pm 0,6)$ y Braford ${ }^{30}(7,0 \pm 1,7)$, utilizando para estos ensayos $260 \mathrm{mg}$ de Folltropin-V en dosis decrecientes cada $12 \mathrm{~h}$, durante 4 días.

Los embriones degenerados $(1 \pm 0,12)$ resultaron inferiores a los informados, de 3,5 trabajando con FSH-P: 3 mg IM dos veces al día durante 5 días ${ }^{4}$. Los ovocitos sin fertilizar $(5,32 \pm 1,01)$ se mostraron superiores a los declarados, de 3,19 $\pm 3,09$, en Brahman con un protocolo P-24 similar al T2 ${ }^{23}$.

En la Tabla 1 se presenta la estadística descriptiva para el número de cuerpos lúteos en ovario izquierdo, derecho y totales según T1 y T2 en donantes Braford del nordeste argentino (NEA).

La ultrasonografía no mostró diferencias en la cantidad de cuerpos lúteos totales, sin embargo, fue más alto en el ovario derecho respecto del izquierdo. Los

Tabla 1: Media aritmética para el número de cuerpos lúteos en ovarios izquierdos (izq), derechos (der) y totales (tot) según T1 y T2 .

\begin{tabular}{lccc}
\hline tratamiento & izq & der & tot \\
\hline T1 (día 3) & 5,6 & 7,0 & 12,6 \\
T2 (día 4) & 5,4 & 6,66 & 12,06 \\
\hline
\end{tabular}


Tabla 2. Frecuencias absolutas y relativas de estructuras ováricas: cuerpo lúteo (CL), folículo dominante (FD), folículos de 1 a $3 \mathrm{~mm}$ (Fol 1-3 mm), de 3 a $6 \mathrm{~mm}$ (Fol 3-6 mm) y totales; prueba de chi $^{2}$ y p-valor, según T1 y T2 en los momentos de las ultrasonografías, en donantes Braford del NEA.

\begin{tabular}{|c|c|c|c|c|c|c|}
\hline momento & estructura & $\mathrm{T} 1$ & $\mathrm{~T} 2$ & total & $\chi^{2}$ & $\mathrm{p}$-valor \\
\hline \multirow{5}{*}{$\mathrm{P} 4+17-\beta \mathrm{e}+\mathrm{CIDR}$} & $\mathrm{CL}$ & $8(0,07)$ & $9(0,09)$ & $17(0,08)$ & \multirow{5}{*}{0,65} & \multirow{5}{*}{0,8842} \\
\hline & FD & $11(0,10)$ & $9(0,09)$ & $20(0,10)$ & & \\
\hline & Fol 1-3mm & $64(0,58)$ & $51(0,54)$ & $115(0,56)$ & & \\
\hline & Fol 3-6mm & $27(0,25)$ & $26(0,27)$ & $53(0,26)$ & & \\
\hline & Total & $110(1,0)$ & $95(1,0)$ & $205(1,0)$ & & \\
\hline \multirow{5}{*}{ Inicio de FSH } & $\mathrm{CL}$ & $8(0,07)$ & $10(0,09)$ & $18(0,08)$ & \multirow{5}{*}{1,89} & \multirow{5}{*}{0,5947} \\
\hline & FD & $3(0,03)$ & $3(0,03)$ & $6(0,26)$ & & \\
\hline & Fol 1-3mm & $66(0,55)$ & $64(0,60)$ & $110(0,49)$ & & \\
\hline & Fol 3-6mm & $42(0,35)$ & $29(0,27)$ & $71(0,31)$ & & \\
\hline & Total & $119(1,0)$ & $106(1,0)$ & $225(1,0)$ & & \\
\hline \multirow{5}{*}{ celo } & $\mathrm{CL}$ & $0(0,00)$ & $2(0,01)$ & $2(0,01)$ & \multirow{5}{*}{5,27} & \multirow{5}{*}{0,1528} \\
\hline & FD & $78(0,44)$ & $109(0,53)$ & $187(0,49)$ & & \\
\hline & Fol 1-3mm & $64(0,36)$ & $62(0,30)$ & $126(0,33)$ & & \\
\hline & Fol 3-6mm & $34(0,19)$ & $31(0,15)$ & $65(0,17)$ & & \\
\hline & Total & $176(1,0)$ & $204(1,0)$ & $380(1,0)$ & & \\
\hline \multirow{4}{*}{ colecta } & $\mathrm{CL}$ & $100(1,00)$ & $109(0,98)$ & $209(0,99)$ & \multirow{4}{*}{1,82} & \multirow{4}{*}{0,4027} \\
\hline & FD & $0(0,00)$ & $1(0,01)$ & $1(4,7 \mathrm{E}-03)$ & & \\
\hline & Fol 1-3mm & $0(0,00)$ & $1(0,01)$ & $1(4,7 \mathrm{E}-03)$ & & \\
\hline & Total & $100(1,0)$ & $111(1,0)$ & $211(1,0)$ & & \\
\hline
\end{tabular}

resultados fueron superiores a los generados en otras investigaciones, de $8,8 \pm 1,7$ en vacas tratadas con aspiración del folículo dominante previo al tratamiento superovulatorio ${ }^{10}$ y de $8,0 \pm 1,5$ y $7,0 \pm 1,4$ en vaquillas y vacas respectivamente, tratadas con eCG y anti eCG (Folligon ${ }^{\circledR}$ 2500IU IM y Neutra-PMSG ${ }^{\circledR} 5 \mathrm{ml}$ ) ${ }^{18}$.

En la Tabla 2 se exhiben los resultados de la prueba de $\operatorname{chi}^{2}$ y las frecuencias absolutas y relativas de las estructuras ováricas estudiadas por ultrasonografía.

Mediante la prueba de independencia no se pudo establecer asociación entre tratamientos y estructuras ováricas, en ninguna de las ultrasonografías.

Los resultados de dicha técnica en el día 0 para folículos de 1 a $3 \mathrm{~mm}$ tanto en $\mathrm{T} 1$ como en T2, fueron inferiores a $95,12 \%$ en vacas anéstricas superovuladas pretratadas con implantes de Syncro-Mate-B y benzoato de estradiol en otros trabajos ${ }^{20}$. Para folículos de 3 a 6 $\mathrm{mm}$ los resultados de $\mathrm{T} 1$ y T2 se mostraron superiores a los informados por los mismos autores ${ }^{20}$, de $4,88 \%$, mientras que no registraron folículos dominantes ni cuerpos lúteos, posiblemente debido a que trabajaron con vacas en anestro ${ }^{20}$.

Los folículos de 1 a $3 \mathrm{~mm}$, en el día del inicio de las aplicaciones de FSH, fueron inferiores a 97,04\% y los de 3 a 6 mm superiores a $2,96 \%{ }^{20}$.

El día del celo las estructuras más importantes fueron los folículos dominantes, tanto en T1(13) como en T2 $(18,16)$; superiores a $5 \pm 1,2$ en vacas Holstein utilizando FSH (Folltropin, Vetrepharm, Canadá) 6 días después de sincronizar la onda folicular con $6 \mathrm{mg}$ de Norgestomet y $5 \mathrm{mg}$ de Valerato de Estradiol ${ }^{22} \mathrm{e}$ inferiores a $20,3 \pm 4,8$ en razas de carne en un protocolo similar $^{6}$.
En el día de la colecta, el número de cuerpos lúteos fue similar entre ambos tratamientos (16,6 vs. 18,16 en $\mathrm{T} 1$ y $\mathrm{T} 2$, respectivamente), superiores a lo informado de $9,9 \pm 1,8$ en vacas Holstein ${ }^{22}$ y similares a $16,6 \pm 3,4$ obtenidos en vacas de carne superovuladas 5 días después de iniciada la onda folicular utilizando $\mathrm{FSH}^{6}$.

Concluimos afirmando que, bajo las condiciones en las que se realizó el ensayo, si bien el adelanto en la superestimulación en donantes de embriones Braford del NEA no generó mejoras en la respuesta a la ultrasonografía, puede ser utilizado como una alternativa en la producción de embriones acortando el tratamiento tradicional y/o por razones de logística, sin afectar la cantidad y calidad de estructuras ováricas y embriones recuperados.

Agradecimientos. Al personal de campo, por su colaboración en los tratamientos y el trabajo de manga. A los Sres. Gerardo Taubas y Daniel Perichón por su apoyo en el laboratorio. A la M.V. Nolly María Monzón y al Dr. Fernando Revidatti por revisar el Abstract de esta publicación.

\section{REFERENCIAS}

1. Adams GP, Jaiswa IR, Singh J, Malhi P. 2008. Progress in understanding ovarian follicular dynamics in cattle. Theriogenology 69: 72-80.

2. Armstrong DT. 1993. Recent advances in superovulation of cattle. Theriogenology 39: 7-24.

3. Baruselli PS et al. 2003. Adequação da dose de FSH (Folltropin-v) em protocolos de superovulação de vacas nelore (Bos Taurus-indicus) com inseminação artificial em tempo fixo (SOTF). Acta Sci Vet 31: 244-245. 
4. Becker WA, Pinheiro LE. 1986. Ovarian response to superovulation in Nelore cows (Bos Taurus-indicus). Theriogenology 25: 785-793.

5. Bó GA, Hockley DK, Nasser LF, Mapletoft RJ. 1994. Superovulatory response to a single subcutaneous injection of a porcine pituitary extract in beef cattle. Theriogenology 42: 963-975.

6. Bó GA, Adams GP, Pierson RA, Mapletoft RJ. 1996. Effect of progestogen plus estradiol-17P treatment on superovulatory response in beef cattle. Theriogenology. 45 : 897-910.

7. Di Rienzo JA, Casanoves F, Balzarini MG, Gonzalez L, Tablada M, Robledo CW. 2011. InfoStat.GrupoInfoStat, FCA, Universidad Nacional de Córdoba, Argentina.

8. Donaldson LE. 1984. The day of the estrus cycle that FSH is started and superovulation in cattle. Theriogenology. 22: 97-99.

9. Elsden R, Hasler J, Seidel G. 1976. Non-surgical recovery of bovine eggs. Theriogenology 6: 523-532.

10. Foote RH. 2003. Fertility estimation: a review of past experience and future prospects. Anim Reprod Sci 75: 119139.

11. Gorlach A. 1999. Transferencia de embriones en ganado vacuno, 1ra.ed., Ed. Acribia, Zaragoza, España, 355 p.

12. Greve T, Lehn H, Fasbacho NO. 1977. Non surgical recovery of bovine embryos. Theriogenology. 7: 239-251.

13. Hasler JF. 1992. Current status and potential of embryo transfer and reproductive technology in dairy cattle. $J$ Dairy Sci 75: 2857-2879.

14. Henao RG. 2010. Some factors related to follicular dynamics in Bos indicus. Rev Fac Nal Agr Medellín, Colombia, 63: 5577-5586.

15. Huhtinen M, Rainio V, Aalto J, Bredbacka P, Maki. 1992. Increased ovarian responses in the absence of a dominant follicle in superovulated cows. Theriogenology 37: 457-463.

16. Jiménez C. 2009. Superovulación: estrategias, factores asociados y predicción de la respuesta superovulatoria en bovinos. Rev Med Vet Zoot 56: 195-214.

17. Kanagawa H, Shimohira I, Saitoh N. 1995. Manual of bovine embryo transfer, Livestock Technology Association, Japan, $432 \mathrm{p}$.

18. Lopes L, Chagas J, Robalo J. 2001. Superovulatory response, embryo quality and fertility after treatment with different gonadotrophins in native cattle. Theriogenology 56: 65-77.
19. Monniaux D, Chupin D, Saumande J. 1983. Superovulatory responses of cattle. Theriogenology 19: 55-81.

20. Morales C, Moreira OL, Bernal A, Faure R. 2001. Respuesta superovulatoria en vacas anéstricas pre-tratadas con implantes de Syncro-mate-B y benzoato de estradiol. Rev Salud Anim 23: 187-199.

21. Ochoa JC, Ramirez RR, Piccardi MB, Bó GA, Tríbulo H. 2009. Influencia en la estación en la producción de embriones en donantes de embriones de raza para carne. VIII Simposio Internacional de Reproducción Bovina, Córdoba, Argentina. http://www.iracbiogen.com.ar/front/biblioteca. asp? Modo $=$ Leer $\& I D=95$

22. Oropeza A, Bastidas P. 2003. Efecto del norgestomet y el valerato de estradiol sobre la actividad ovárica en vacas Holstein. Gaceta de Ciencias Veterinarias 8: 51-60.

23. Salgado OR, Mejía AA, Suarez PS. 2011. Eficiencia de la respuesta superovulatoria del ganado Brahman al protocolo p-24. Rev Med Vet Zoot Córdoba 16: 2521-2527.

24. Savio JD, Keenan L, Boland MP, Roche JF. 1988. Pattern of growth of dominant follicles during the oestrous cycle of heifers. J Reprod Fertil 83: 663-671.

25. Stringfellow DA, Seidel SM. 1998. Manual of the International Embrio Transfer Society, $3^{\text {rd }}$ ed., Ed. Savoy, Illinois, USA, $170 \mathrm{p}$.

26. Sutherland W. 1991. Biomaterials. Novel material from biological sources, $1^{\mathrm{a}}$ ed., Stockton Press, New York, USA, p.307-333.

27. Tríbulo A et al. 2009a. Efecto de la dosis y protocolo de administración de Folltropin-V en la producción de embriones de donantes Angus. Anales VIII Simposio Intern. Reprod. Bov., Córdoba, Argentina.

28. Tríbulo A et al. 2009b. Efecto de la dosis y protocolo de administración de Folltropin-V en la producción de embriones de donantes Bonsmara. Anales VIII Simposio Intern. Reprod. Bov., Córdoba, Argentina.

29. Tríbulo A et al. 2009c. Efecto de la dosis y protocolo de administración de Folltropin- $\mathrm{V}$ en la producción de embriones de donantes Brangus. Anales VIII Simposio Intern. Reprod. Bov., Córdoba, Argentina.

30. Tríbulo A et al. 2009d. Superovulación de donantes Braford con una dosis de Folltropin-V. Anales VIII Simposio Intern. Reprod Bov., Córdoba, Argentina. 